\title{
Performance evaluation of finite-source Cognitive Radio Networks with impatient customers
}

\author{
Mohamed Hedi Zaghouani, János Sztrik \\ Doctoral School of Informatics, University of Debrecen, Debrecen, Hungary \\ zaghoueni.hedi@gmail.com, sztrik.janos@inf.unideb.hu \\ Submitted: February 4, 2020 \\ Accepted: July 1, 2020 \\ Published online: July 23, 2020
}

\begin{abstract}
The current paper takes into consideration a cognitive radio network with impatient customers, by the help of finite-source retrial queueing system. We consider two different types of customers (Primary and Secondary) assigned to two interconnected frequency bands. A first frequency band with a priority queue and a second one with an orbit, both are respectively dedicated for the Primary Users (PUs) and Secondary Users (SUs). In case the servers are busy, both customers (Licensed and Unlicensed) join either the queue or the orbit. Before joining the orbit, secondary customers receive a random retrial time according to Exponential distribution, which is the holding time before the next retry. Unlicensed users (impatient) are obliged to leave the system once their total waiting time exceeds a given maximum waiting time.

The novelty of this work is the investigation of the abandonment and its impact on several performance measures of the system such as the mean response time and waiting time of users, probability of abandonment of SU, etc. Several figures illustrate the problem in question by the help of simulation.

Keywords: Retrial queuing systems, simulation, cognitive radio networks, performance and reliability measures, Impatient customers, Tandem queue, Abandonment.
\end{abstract}




\section{Introduction}

Cognitive Radio (CR) is a smart technique capable to get rid of the under-utilization spectrum issues, by allowing secondary customers to use opportunistically the primary channel without performing any interference to the communication of the primary customers to enhance the performance of the network. This intelligent technology is skilled to modify its transmitter parameters in compliance with the interaction of the environment in which it operates. The main objective of CRN is to exploit the unused sections of the primary frequency bands for the favour of unlicensed customers, more details can be found in [1, 6, 8, 24].

The expression cognitive radio was presented for the first time in 1999 by Mitola [13], explaining that this technology is conscious of the surrounding environment where performers and can adjust its parameters to improve customers performance. Several studies and researches, as [25, 28] reveal that often many parts of the channels are unused in time and space. These parts (white spaces) are not occupied by any licensed users. Secondary users in these parts of the service unit can detect this disuse and communicate freely with each other without performing any harmful consequences on the primary users. Nowadays, two types of Cognitive Radio Network exist. The first type is termed as (underlay network) in which unlicensed users might use the primary channels simultaneously with the licensed users, based on some predetermined conditions. The second type is called (overlay networks) where the unlicensed users are allowed at any given time to use the Primary Service Unit whilst it is unoccupied by licensed customers, more information was introduced by the authors of $[18,22,26,29]$. However, the current paper deals with overlay $\mathrm{CRN}$, by modelling a system that contains two finite-source subsystems(Primary and Secondary).

In this queuing system, we take into account two elements, a first subsystem is allocated for the jobs of Primary Users (PU), with a finite number of sources. In this subsystem, each source generates a primary call for the PUs after an exponentially distributed time, the latter requests are forwarded to a single server Primary Channel Service (PCS) with a preemptive discipline (FIFO queue) to start the service, supposing that the service time is exponentially distributed as well. The second component of the model is built for the requests of Secondary Users (SU), coming from a fi nite-source and heading to the Secondary Channel Service (SCS), knowing that the source and service times of the secondary users are exponentially distributed.

The generated primary tasks are targeting the PCS to check its accessibility. If this service unit is unoccupied, the service starts immediately. However, if the PCS is busy by another primary job, this last task joins a First In First Out (FIFO) queue. Nevertheless, if a secondary job is being treated in the primary unit, this job disconnects right away and should be routed back to the Secondary Channel Service.

Per the status of the secondary channel, the cancelled task either begins again the service on its original server (SCS) or joins the retrial queue (orbit). Besides, 
the secondary channel will receive the low priority requests. If the aimed unit is idle, the service might start immediately, otherwise, these secondary requests will try to join the primary unit. If it is free the secondary requests have the opportunity to begin. If not, they will join automatically the orbit. From the orbit, the postponed requests retry to get the service after an exponentially distributed random interval, more details can be found in $[6,8,18,22,24,29]$.

In this study, we assume that impatient customers in the orbit who their total waiting times exceed a given maximum waiting time need to leave the system, which is the novelty of this work.

Several studies have investigated the CRN based on different scenarios. Taking [20] as an example the authors have used some methods of queuing theoretical on a finite source cognitive radio network with two service units (primary and secondary) in order to examine the main performances of this system by the help of tool-supported approach.

However, in a similar work [2] authors studied a single server network, which is subject to breakdowns and repairs. This kind of networks suffer from many difficulties while dealing with the requests, as the breakdown of the only server affects the whole network.

By the help of retrial queuing model and using Primary and Secondary service channels [14] supposed that both units are subject to random breakdowns and repairs. The authors used several distributions (Exponential, Hypo and Hyper Exponential) in order to show the impact of these distributions on the performance measures of the system.

As extended work, in [27] Gamma distribution was added to the above mentioned above distributions.

In papers [15] and [16] authors used as well Hypo, Hyper and Exponential distributions supposing that the secondary users of the system are subject to collisions and the two services in the system are unreliable, respectively.

However, after a deep dive in many similar investigations and studies, we figured out that none of them dealt with this model taking into consideration the abandonment phenomena.

Instead, many probes analysed abandonment in other types of network, endorsing that customers can leave systems from queues, server units, while getting services and while waiting, more details are found in [5, 9-11, 23]. However, in the present paper, we suppose that the impatient users (Secondary ones) are forced to leave the system from the orbit only while waiting.

Several figures will show the impact of the abandonment on the performance measures of the system, by the help of simulation.

\section{System's operation model}

Fig.1 demonstrates a finite source queuing system that models the considered cognitive radio network. Our queuing system consists of two not independent, interconnected sub-systems. A first part is allocated to primary requests, with $N 1$ the 


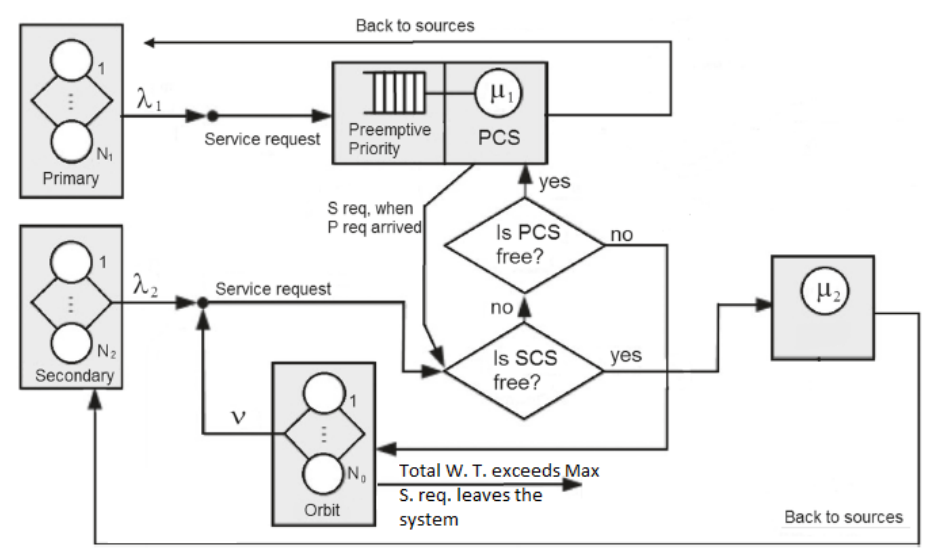

Figure 1: Finite-source retrial queuing system: Modeling the Cognitive Radio Network with impatient customers

number of sources. These sources will be responsible for generating a high priority requests with an inter-request time exponentially distributed, using a parameter $\lambda_{1}$. All the produced requests are directed to a single server unit (PCS) with a FIFO queue. The service times of the primary tasks are supposed to be exponentially distributed as well with rate $\mu_{1}$.

The second subsystem is devoted for the low-priority requests. The number of sources is denoted by $N 2$, the inter-arrival times and service times in this subsystem are assumed to be exponentially distributed with parameter $\lambda_{2}$ and $\mu_{2}$, respectively.

Both servers can be in two states: idle or busy. Per the server's state, the generated primary packet goes to the primary server (if the server is idle) or joins the FIFO queue (if the service unit is busy with a PU). However, if the PCS is occupied by an unlicensed user, its service is instantly stopped and the interrupted secondary request is sent back to the Secondary unit.

Depending on the availability of the secondary unit, the aborted task is addressed either to the server or the retrial queue (orbit) and reties again its service from the beginning after an exponentially distributed time with parameter $\nu$.

In the other hand, requests from SUs are directed to SCS. If it is idle, the service begins, if not, this unlicensed task will sens the PCS. In case of an idle status for PCS, this service may opportunistically join the high priority channel. If the PCS is engaged, the request goes to the orbit. It should be noted that Secondary Users in the orbit are obliged to leave the system once their total waiting time will exceed a specified maximum waiting time.

We introduce the following notations, to create a stochastic process describing the behaviour of the system:

- $k 1(t)$ : represents the number of licensed sources at given time $t$;

- $k 2(t)$ : refers to the number of unlicensed at time given $t$; 
- $q(t)$ : is the number of primary requests in the queue at certain time $t$;

- $o(t)$ : denotes the number of tasks in the orbit at time $t$;

- $y(t)=0$, if the primary channel is idle, $y(t)=1$, if the primary channel is processing (busy) a high-priority request and $y(t)=2$, if the primary service unit is processing (busy) a low-priority request at time $t$;

- $c(t)=0$, if the secondary service unit is idle(free) and $c(t)=1$, if the secondary service unit is busy at given time $t$.

As consequence we can see that:

$$
k 1(t)= \begin{cases}N 1-q(t), & \mathrm{y}(\mathrm{t})=0,2, \\ N 1-q(t)-1, & \mathrm{y}(\mathrm{t})=1 .\end{cases}
$$

As a result we can see that:

$$
k 2(t)= \begin{cases}N 2-o(t)-c(t), & \mathrm{y}(\mathrm{t})=0,1, \\ N 2-o(t)-c(t)-1, & \mathrm{y}(\mathrm{t})=2 .\end{cases}
$$

We assume that all the random variables used in the model construction are exponentially distributed, therefore we decided to use a stochastic simulation by the help of $\mathrm{C}$ coding language with GSL stochastic library.

All the numerical results of this were collected by the validation of the simulation outputs. Speaking of exponentially distributed inter-event time, we can construct continuous-time Markov chain and the main steady-state performance measures can be obtained, see for example [19]. The input parameters are displayed in Table 1 .

\begin{tabular}{|c|c|c|}
\hline Parameters & Value at moment $t$ & Maximum Value \\
\hline Primary sources & $k 1(t)$ & N1 \\
Secondary Sources & $k 2(t)$ & N2 \\
Primary arrival rate & $\lambda_{1}$ & \\
Secondary arrival rate & $\lambda_{2}$ & N1-1 \\
Number of requests at the queue (FIFO) & $q(t)$ & N2-1 \\
Number of requests at the orbit & $o(t)$ & \\
Primary service rate & $\mu_{1}$ & \\
Secondary service rate & $\mu_{2}$ & \\
\hline
\end{tabular}

Table 1: Parameters of the simulation

\section{Simulation results}

The batch-mean method was used in the simulation to estimate the mean response times of the requests. This method is a common confidence interval technique 
which is applied for steady-state simulation output analysis. See for example [3, 4, $7,12,21]$.

Based on the values shown in Table 2 we could generate several figures supposing that the maximum waiting time of $\mathrm{SU}$ is constant, it should be noted as well, that in our simulation the Secondary Users were divided into two categories (Successful and Abandoned).

\begin{tabular}{|l|l|l|l|l|l|l|l|l|}
\hline Figure No. & $\mathrm{N} 1$ & $\mathrm{~N} 2$ & $\lambda_{1}$ & $\lambda_{2}$ & $\mu_{1}$ & $\mu_{2}$ & $\nu$ & MaxW.T. \\
\hline Figure 2,3 & 7 & 12 & 0.5 & 0.2 & 2 & 1 & 10 & x-axis \\
\hline Figure 4 & 6 & 6 & 0.6 & $\mathrm{x}$-axis & 4 & 4 & 0.4 & 10 \\
\hline Figure 5 & 8 & 10 & 0.5 & 0.2 & 1 & 0.5 & 20 & x-axis \\
\hline
\end{tabular}

Table 2: Numerical values of model parameters

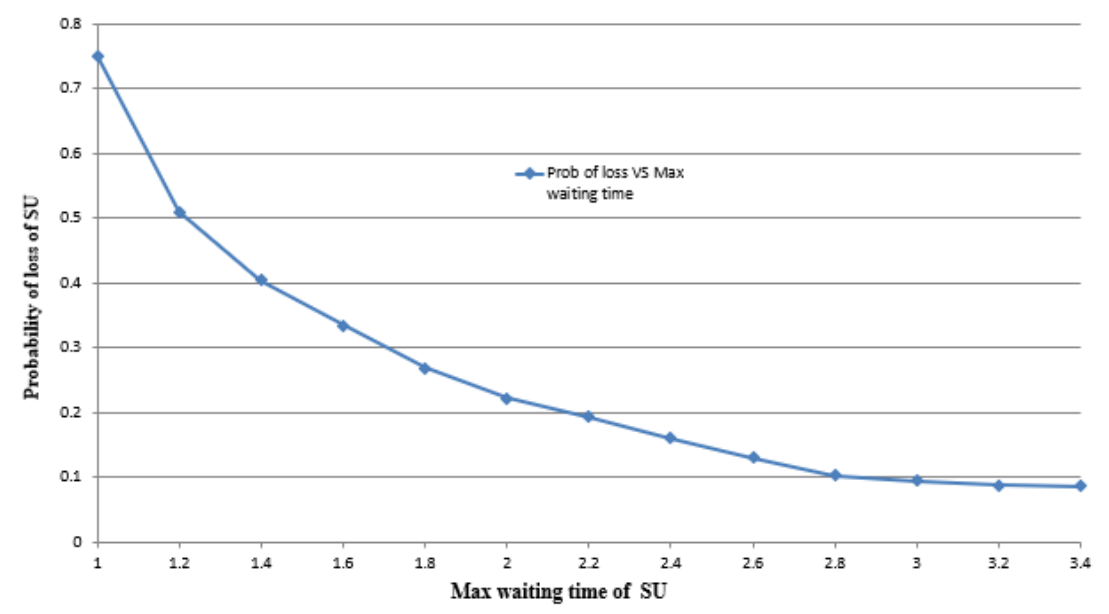

Figure 2: The effect of the Maximum waiting time of SU on the Probability of loss of SU

Figure 2 illustrates the impact of the maximum waiting time of SU on the probability of loss, as anticipated, by increasing the abandonment time, the probability of loss decreases as more secondary customers have the chance to get served without leaving the system, which makes the SCS busier.

Using the following formula we could generate Figure 3:

$$
\overline{W_{a}}=P_{a b o n} . C+\left(1-P_{a b o n}\right) \overline{W_{\text {succ }}}
$$

- $\overline{W_{a}}$ : Mean waiting time of an arbitrary (patient or impatient) SU

- $P_{a b o n}$ : Probability of abandonment

- $C$ : Maximum waiting time of SU 


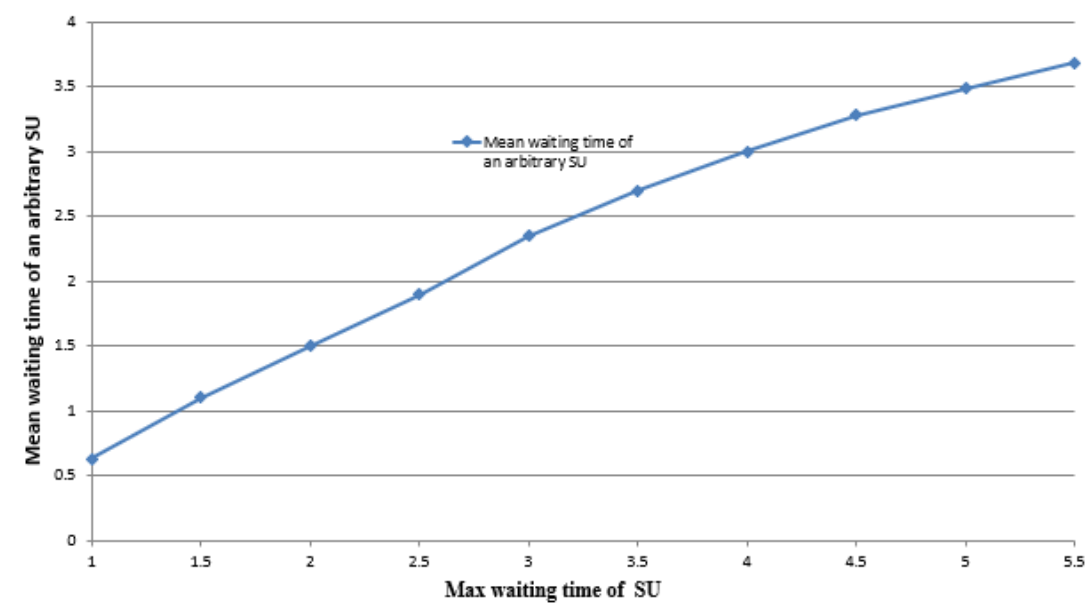

Figure 3: The effect of the Maximum waiting time of SU on the mean waiting time of an arbitrary SU

- $\overline{W_{\text {succ }}}$ : Mean waiting time of successful Secondary user.

In Figure 3 the effect of abandonment time of SU on the mean waiting time of an arbitrary SU (Patient or Impatient) was displayed. This figure confirms the expectation that is increasing the maximum waiting time for $\mathrm{SU}$ involves higher waiting times for the two categories of unlicensed customers.

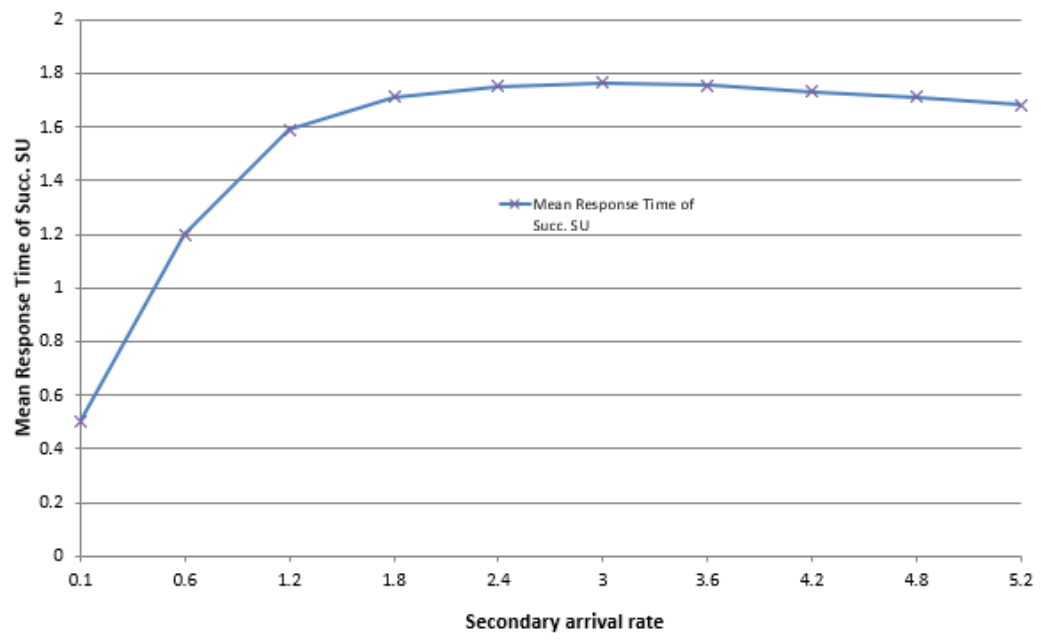

Figure 4: The effect of the secondary arrival rate on the mean response time of the successful Secondary Users 
Figure 4 shows the effect of the request generation rate on the mean response time of the secondary users. The result presents the phenomenon of having a maximum value of the mean response time which was noticed in [17]. The abandonment of impatient SU from the orbit provides shorter response time for the patient users.

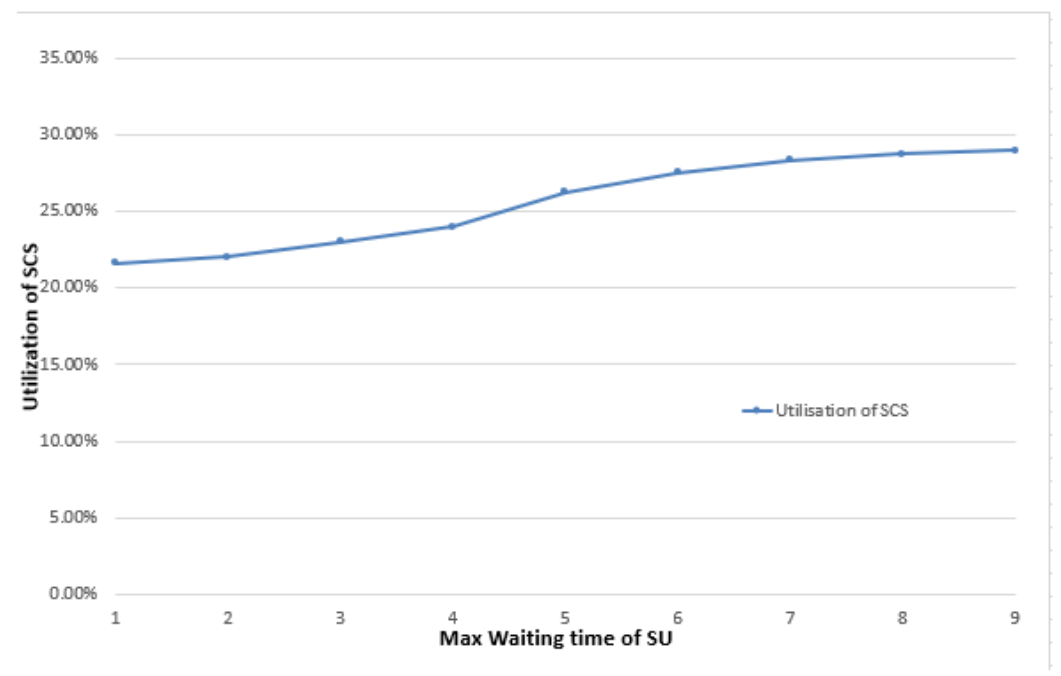

Figure 5: The effect of the Maximum waiting time of SU on the Utilization of SCS

The last Figure exhibits the effect of the abandonment time of the secondary users on the utilization of the secondary server. The innovation of abandonment contributes less utilization for the server when the maximum waiting time is too small, as a consequence, only the SU with a small amount of waiting time will benefit the service.

\section{Conclusion}

In this paper a finite-source retrial queuing model was introduced using two not independent, interconnected channels servicing licensed and unlicensed users in a cognitive radio network with abandonment from the orbit. Licensed users have preemptive priority over the unlicensed ones in servicing at the primary channel. However, at the secondary channel, an orbit was established for the secondary jobs finding the secondary service unit occupied upon arrival. SU may leave the system from the orbit, once their total waiting time reaches a given maximum. By the help of simulation, several sample examples were obtained, showing the effect of the abandonment on the different performance measures of the system.

Lastly, as future work, we will keep investigating the impact of the abandon- 
ment on a such system assuming that the maximum waiting time is a generally distributed random variable.

Acknowledgements. The research work of János Sztrik and Mohamed Hedi Zaghouani is supported by the construction EFOP-3.6.3-VEKOP-16-2017-00002 and by the Stipendium Hungaricum Scholarship, respectively.

\section{References}

[1] I. F. Akyildiz, W.-Y. Lee, M. C. Vuran, S. Mohanty: Next generation/dynamic spectrum access/cognitive radio wireless networks: A survey, Computer networks 50.13 (2006), pp. $2127-2159$, DOI: https://doi.org/10.1016/j.comnet.2006.05.001.

[2] B. Almási, T. Bérczes, A. Kuki, J. Sztrik, J. Wang: Performance modeling of finitesource cognitive radio networks, Acta Cybernetica 22.3 (2016), pp. 617-631, DOI: https://doi.org/10.14232/actacyb.22.3.2016.5.

[3] E. Carlstein ET AL.: The use of subseries values for estimating the variance of a general statistic from a stationary sequence, The annals of statistics 14.3 (1986), pp. 1171-1179, DOI: https://doi.org/10.1214/aos/1176350057.

[4] E. J. Chen, W. D. KeLton: A procedure for generating batch-means confidence intervals for simulation: Checking independence and normality, Simulation 83.10 (2007), pp. 683-694, DOI: https://doi.org/10.1177/0037549707086039.

[5] E. Danilyuk, O. Vygoskaya, S. Moiseeva: Retrial queue $M / M / N$ with impatient customer in the orbit, in: International Conference on Distributed Computer and Communication Networks, Springer, 2018, pp. 493-504, DOI: https://doi.org/10.1007/978-3-319-99447-5_42.

[6] N. Devroye, M. Vu, V. Tarokn: Cognitive radio networks, IEEE Signal Processing Magazine 25.6 (2008), pp. 12-23, DOI: https://doi.org/10.1109/MSP. 2008.929286.

[7] G. S. Fishman, L. S. Yarberry: An implementation of the batch means method, INFORMS Journal on Computing 9.3 (1997), pp. 296-310, DOI: https://doi.org/10.1287/ijoc.9.3.296.

[8] S. Gunawardena, W. Zhuang: Modeling and Analysis of Voice and Data in Cognitive Radio Networks, Springer, 2014, DOI: https://doi.org/10.1007/978-3-319-04645-7.

[9] Q.-M. He, H. Zhang, Q. Ye: An $M / P H / K$ queue with constant impatient time, Mathematical Methods of Operations Research 87.1 (2018), pp. 139-168, DOI: https://doi.org/10.1007/s00186-017-0612-2.

[10] R. Ibrahim: Managing queueing systems where capacity is random and customers are impatient, Production and Operations Management 27.2 (2018), pp. 234-250, DOI: https://doi.org/10.1111/poms.12796.

[11] R. Kulshrestha ET AL.: Channel allocation and ultra-reliable communication in CRNs with heterogeneous traffic and retrials: A dependability theory-based analysis, Computer Communications (2020).

[12] A. M. Law, W. D. Kelton, W. D. Kelton: Simulation modeling and analysis, vol. 3, McGraw-Hill New York, 2000.

[13] J. Mitola, G. Q. MaguiRe: Cognitive radio: making software radios more personal, IEEE personal communications 6.4 (1999), pp. 13-18, DOI: https://doi.org/10.1109/98.788210. 
[14] H. Nemouchi, J. Sztrik: Performance Simulation of Finite-Source Cognitive Radio Networks with Servers Subjects to Breakdowns and Repairs, Journal of Mathematical Sciences 237.5 (2019), pp. 702-711, DOI: https://doi.org/10.1007/s10958-019-04196-y.

[15] H. Nemouchi, J. Sztrik: Performance evaluation of finite-source cognitive radio networks with collision using simulation, in: 2017 8th IEEE International Conference on Cognitive Infocommunications (CogInfoCom), IEEE, 2017, pp. 000127-000131, DOI: https://doi.org/10.1109/CogInfoCom.2017.8268228.

[16] H. Nemouchi, J. Sztrik: Performance evaluation of finite-source cognitive radio networks with non-reliable services using simulation, in: Annales Mathematicae et Informaticae, vol. 49, Eszterházy Károly University Institute of Mathematics and Informatics, 2018, pp. 109-122, DOI: https://doi.org/10.33039/ami.2018.12.001.

[17] H. Nemouchi, J. Sztrik: Performance Simulation of Non-reliable Servers in Finite-Source Cognitive Radio Networks with Collision, in: International Conference on Information Technologies and Mathematical Modelling, Springer, 2017, pp. 194-203, DOI: https ://doi.org/10.1007/978-3-319-68069-9_16.

[18] F. Palunčić, A. S. Alfa, B. T. Maharaj, H. M. Tsimba: Queueing models for cognitive radio networks: A survey, IEEE Access 6 (2018), pp. 50801-50823, DOI: https://doi.org/10.1109/ACCESS.2018.2867034.

[19] J. Sztrik: On the finite-source $G / M / r$ queue, European Journal of Operational Research 20.2 (1985), pp. 261-268, DOI: https://doi.org/10.1016/0377-2217(85)90068-2.

[20] J. Sztrik, B. Almási, J. Roszik: Heterogeneous finite-source retrial queues with server subject to breakdowns and repairs, Journal of Mathematical Sciences 132.5 (2006), pp. 677685 ,

DOI: https://doi.org/10.1007/s10958-006-0014-0.

[21] H. C. Tijms: A first course in stochastic models, John Wiley and sons, 2003, DOI: https://doi.org/10.1002/047001363X.

[22] T. Van Do, N. H. Do, Á. Honváth, J. Wang: Modelling opportunistic spectrum renting in mobile cellular networks, Journal of Network and Computer Applications 52 (2015), pp. 129138 , DOI: https://doi.org/10.1016/j.jnca.2015.02.007.

[23] J. Wang, H. Abouee Mehrizi, O. Baron, O. Berman: Staffing Tandem Queues with Impatient Customers-Application in Financial Service Operations, Rotman School of Management Working Paper 3116815 (2018), DOI: https://doi.org/10.2139/ssrn.3116815.

[24] L. Wang, C. WAng, F. Adachi: Load-Balancing Spectrum Decision for Cognitive Radio Networks, IEEE Journal on Selected Areas in Communications 29.4 (2011), pp. 757-769, DOI: https://doi.org/10.1109/JSAC.2011.110408.

[25] T. A. Weiss, F. K. Jondral: Spectrum pooling: an innovative strategy for the enhancement of spectrum efficiency, IEEE communications Magazine 42.3 (2004), S8-14, DOI: https://doi.org/10.1109/MCOM.2004.1273768.

[26] E. W. Wong, C. H. Fон: Analysis of cognitive radio spectrum access with finite user population, IEEE Communications Letters 13.5 (2009), pp. 294-296, DOI: https://doi.org/10.1109/LCOMM. 2009.082113.

[27] M. H. Zaghouani, J. Sztrik, A. Uka: Simulation of the performance of Cognitive Radio Networks with unreliable servers, in: Annales Mathematicae et Informaticae.

[28] S. A. ZEKavat, X. Li: User-central wireless system: ultimate dynamic channel allocation, in: First IEEE International Symposium on New Frontiers in Dynamic Spectrum Access Networks, 2005. DySPAN 2005. IEEE, 2005, pp. 82-87. 
[29] Y. Zнао, L. BAI: Performance analysis and optimization for cognitive radio networks with classified secondary users and impatient packets, Mobile Information Systems 2017 (2017), DOI: https://doi.org/10.1155/2017/3613496. 